\title{
Clinical Features and Prognostic Outcome of
}

\section{Renal Collecting Duct Carcinoma: 12 Cases from a Single Institution}

This article was published in the following Dove Press journal: Cancer Management and Research

\author{
Xiaoyuan Qian' \\ Zhixian Wang' \\ Jiaqiao Zhang' \\ Qing Wang' \\ Peng Zhou' \\ Shaogang Wang (D) \\ Bo Wang' \\ Can Qian ${ }^{2}$ \\ 'Department of Urology Surgery, Tongji \\ Hospital, Tongji Medical College, \\ Huazhong University of Science and \\ Technology, Wuhan, People's Republic of \\ China; ${ }^{2}$ Department of Traditional \\ Chinese Medicine and Rheumatology, \\ Southwest Hospital, Army Military \\ Medical University, Chongqing, China
}

Correspondence: Shaogang Wang Tongji Hospital, 1095 Jiefang Avenue, Wuhan City, Hubei Province, People's Republic of China

Email sGWANGTJM@I63.com
Purpose: Collecting duct carcinoma (CDC) is extremely rare and has high malignancy and poor prognosis. The purpose of this research is to explore the clinical characteristic, imaging, pathological diagnosis, treatment and prognostic outcome of CDCs.

Materials and Methods: The clinical data of 12 CDC cases who had been surgically treated between August 2007 and August 2017 and verified the diagnosis of CDC by postoperative pathological and/or immunohistochemical staining (IHC) results were retrospectively analyzed, and related works of literature were reviewed. And Kaplan-Meier survival analysis was used to draw the survival curve and to calculate the survival rate and the median survival time.

Results: According to the TNM stage system, 4 cases were in stage I, 2 in stage II, 3 in stage III, and 3 in stage IV. On the computed tomograph and magnetic resonance imaging, CDC displayed that various shapes, unclear boundary and invasive growth into the renal parenchyma. Compared with small CDCs which did not change the contour of the kidney, large CDCs presented various imaging features. Microscopically, the typical morphology of CDCs was that collecting ducts or tubules were obviously infiltrated by tumor cells. A tubular, papillary, tubulopapillary or solid architectures with desmoplasia were often presented. And tumor cells had high-grade cytology or an infiltrative growth pattern. Necrosis of tumor cells also was common in many cases. The expression of biomarkers, such as PAX-8, INI-1, 34ßE12, CK19, PAX-2, and vimentin, in most patients was detected by IHC. Eleven of all 12 cases received radical surgery, of whom 5 patients died 3-11 months after surgery, and 1 case having undergone interventional embolization therapy died at 6 months after treatment due to multiple metastases. And 1 lost to contact. The overall 1-, 2-, and 5-year survival rates were $45.5 \%, 36.4 \%$, and $8.8 \%$, respectively, and the median survival time (MST) was 11 months. Conclusion: $\mathrm{CDC}$ has an aggressive clinical course, with a poor prognosis. The best way to treat $\mathrm{CDC}$ suspected by imaging examinations is radical surgery which can contribute to confirm the correct histopathological type. And post-operation follow-up is necessary.

Keywords: collecting duct carcinoma, clinical characteristics, imaging, pathology, outcome

\section{Introduction}

Collecting duct carcinoma (CDC) is one of the rare pathological subtypes of renal cell carcinoma (RCC), with high malignancy and poor prognosis. The majority of patients present metastatic carcinoma features at first time diagnosis and have an advanced TNM stage. ${ }^{1,2}$ There is a high incidence of early mortality in which 60 to $70 \%$ of patients died within 3 years of being diagnosed. ${ }^{3}$ Thanks to the rarity of cases of this renal tumor, referential researches are limited. And the clinical 
features, imaging investigations, treatment, and prognosis have been unclear. Currently, the standard treatment for common renal tumors, such as clear-cell renal cell cancer (CCRCC), papillary renal cell cancer (papillary RCC), is well established, but there is no agreement about the optimal treatment strategy for CDCs. In our report, we retrospectively analyzed the clinical, radiologic and pathologic features, treatment, and prognosis outcome of 12 patients with CDC, who had been surgically treated between August 2007 and August 2017. As far as we know, our study includes the largest case number at a single center and may help clinicians to have a good clinical understanding of the CDC.

\section{Materials and Methods \\ Methods}

This retrospective study was approved by the institutional review board (IRB) of Tongji Hospital, Tongji Medical College, Huazhong University of Science and Technology. Because our study belonged to a retrospective study, patient consent to review their medical records was not required by the IRB. And all patient information was strictly confidential and our procedures were carried out according to the Declaration of Helsinki. The study included 12 patients diagnosed with CDC whose data were obtained from August 2007 to August 2017 in the department of Urology Surgery, at Tongji Hospital, Huazhong University of Science and Technology. 1 patient giving up the operation due to old age, large tumor body and multiple co-existing diseases resorted to interventional embolization and the remaining 11 patients accepted surgical treatment in our department whose final diagnosis was determined by the histopathologic assessment. All 12 patients consist of 7 males and 5 females. The clinical presentation, imaging, treatment and prognosis outcomes of 12 patients are presented in Table 1. A urinary CT scan was performed in 9 patients after admission, while MRI in 5 patients. Patients diagnosed with CDC by histopathologic examination were deemed as the inclusion criterion.

\section{Surgical Treatment}

Under general anesthesia,11 patients initially underwent surgery. Retroperitoneal laparoscopic radical nephrectomy (LRN) was performed in 7 cases, and open radical nephrectomy (RN) in 3 cases. And 1 patient giving up due to old age, large tumors and multiple co-existing diseases underwent interventional embolization. At the same time, 1 patient achieved robotic-assisted LRN. Besides, 3 patients were complicated with renal venous tumor thrombus formation, 2 of which were treated with tumor thrombus removal. All operations were completed successfully.

\section{Follow Up}

Clinical and radiographic follow-up information for all patients with CDC were mainly got from outpatient data, added by a telephone interview. The follow-up date spanned from 3-97 months (mean follow-up time, 24months). Follow-up ended on June 1, 2019. The tenth case was out of touch after the initial surgery. The followup rate was up to $91.7 \%$.

\section{Statistical Analysis}

Statistical analysis was performed by using Statistical Product and Service Solutions version 26 (IBM Corporation, Armonk, New York, USA). By using the Kaplan-Meier method, the survival curve was drawn and the median survival time (MST) was calculated.

\section{Results}

\section{Clinical Features}

The demographic features of all patients enrolled in the study are presented in Table 1. In 12 patients, females accounted for $41.7 \%$ and males for $58.3 \%$, with light sex predominance. All cases did not present Hemoglobin abnormalities and family history of hereditary cancers. And only one case was accompanied by a concurrent tubulocystic carcinoma. The age ranged from 30 to $75 y$ ears and the average age was $56.08 \pm 12.98$. The main clinical symptoms included visible hematuria (6 cases $(50.0 \%))$, flank pain (4 cases $(33.3 \%)$ ), asymptomatic ( 2 cases $(16.7 \%))$, fever ( 2 cases $(16.7 \%))$. The disease duration ranged from 2 days to 12 months (mean disease duration, 78 days). The right kidney and the left were involved in $50 \%$ of the patients, respectively, when information about laterality was obtained. According to the TNM stage system, 4 cases were in stage I, 2 in stage II,3 in stage III, and 3 in stage IV.

\section{Imaging results}

A urinary CT scan was performed in 9 patients after admission, while MRI in 5 patients. On CT, the tumor showed slightly lower or middle-density irregular or round soft tissue shadows with clear or unclear borders in most patients. And when enhanced, tumors showed uneven enhancement, and the degree of enhancement was lower than that of peripheral kidney tissues, which was diagnosed as a neoplastic lesion of the kidney (Figure 1). There was 1 case worth noting that 


\begin{tabular}{|c|c|c|c|c|c|c|c|c|c|c|c|c|}
\hline 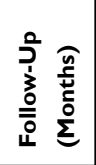 & 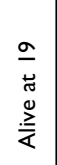 & 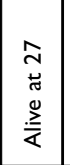 & 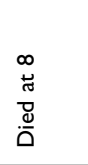 & $\begin{array}{l}\frac{m}{\tilde{m}} \\
\frac{\tilde{y}}{0}\end{array}$ & 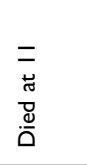 & 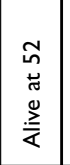 & $\begin{array}{l}\stackrel{0}{0} \\
\tilde{0} \\
\frac{\tilde{y}}{0}\end{array}$ & 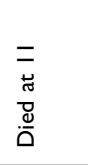 & 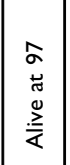 & 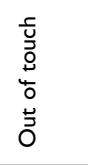 & 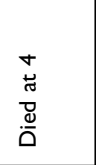 & 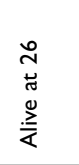 \\
\hline 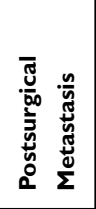 & $\begin{array}{l}\stackrel{\circ}{\frac{0}{5}} \\
\end{array}$ & \begin{tabular}{|c|} 
\\
$\stackrel{0}{0}$ \\
$z$ \\
$z$
\end{tabular} & 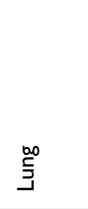 & 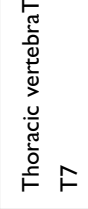 & 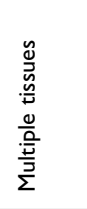 & $\begin{array}{l}\stackrel{0}{5} \\
\stackrel{5}{2}\end{array}$ & 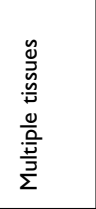 & 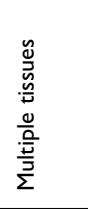 & 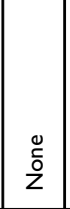 & . & 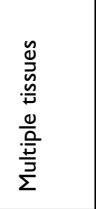 & 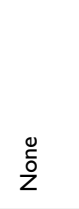 \\
\hline 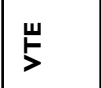 & 号 & \begin{tabular}{|l|}
$\frac{0}{5}$ \\
$\frac{5}{2}$
\end{tabular} & 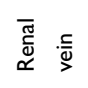 & 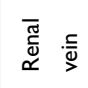 & $\frac{\circ}{\frac{0}{2}}$ & $\begin{array}{ll}\frac{0}{5} \\
\frac{0}{2}\end{array}$ & 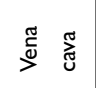 & $\frac{0}{\frac{0}{2}}$ & $\frac{\stackrel{0}{\frac{5}{2}}}{\frac{1}{2}}$ & $\frac{\stackrel{0}{5}}{\frac{5}{2}}$ & 总 & 总 \\
\hline 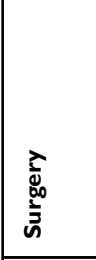 & 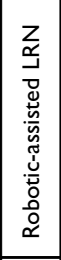 & 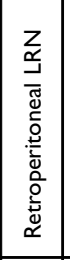 & $\begin{array}{l}z \\
z \\
\text { zon } \\
\text { on } \\
\end{array}$ & $\begin{array}{l}z \\
\text { zo } \\
\text { oğ } \\
\text { on } \\
\end{array}$ & 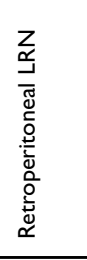 & 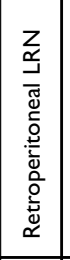 & 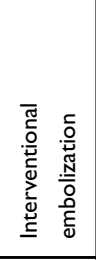 & 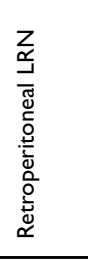 & 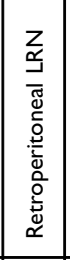 & 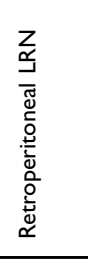 & 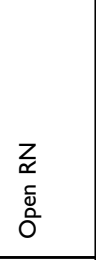 & 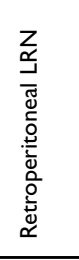 \\
\hline 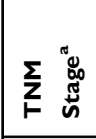 & 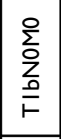 & 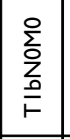 & $\begin{array}{l}\bar{\Sigma} \\
\bar{z} \\
\text { 弟 }\end{array}$ & 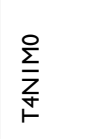 & $\begin{array}{l}\stackrel{0}{0} \\
\stackrel{\sum_{m}^{\prime}}{F}\end{array}$ & 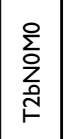 & $\stackrel{\bar{\Sigma}}{\frac{\bar{z}}{4}}$ & 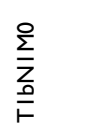 & \begin{tabular}{|l|} 
\\
$\sum_{0}^{0}$ \\
2 \\
$\underline{1}$ \\
1
\end{tabular} & 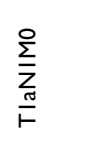 & 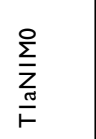 & $\begin{array}{l}\text { 竞 } \\
\text { 䓛 }\end{array}$ \\
\hline 芯 & 符 & \begin{tabular}{|c|c} 
\\
\multirow{i}{*}{} \\
\\
\end{tabular} & 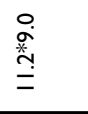 & 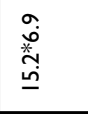 & 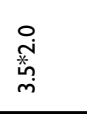 & 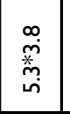 & 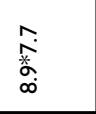 & 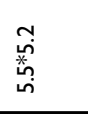 & 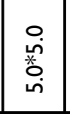 & 䇰 & 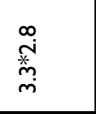 & 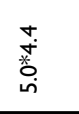 \\
\hline$\stackrel{0}{n}$ & 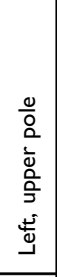 & 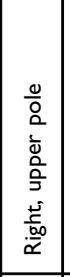 & 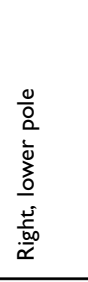 & 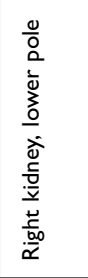 & 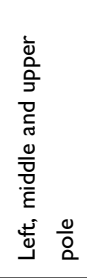 & 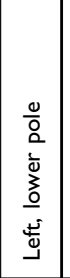 & 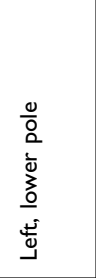 & 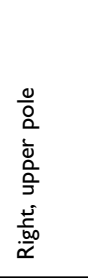 & 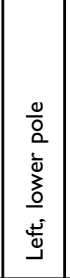 & 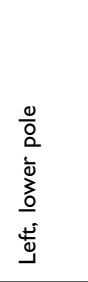 & 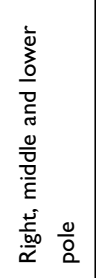 & 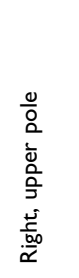 \\
\hline 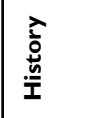 & $\begin{array}{l}\stackrel{\check{g}}{\underline{\Delta}} \\
\stackrel{3}{3}\end{array}$ & 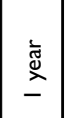 & $\stackrel{\stackrel{\check{\alpha}}{\underline{m}}}{\underline{\underline{y}}}$ & 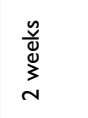 & م) & \begin{tabular}{|l|} 
\\
恿 \\
i
\end{tabular} & 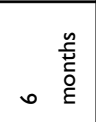 & 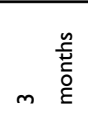 & 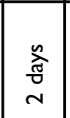 & 产 & 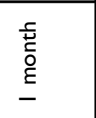 & $\begin{array}{l}\text { 产 } \\
\stackrel{\underline{\sigma}}{\underline{\varepsilon}}\end{array}$ \\
\hline 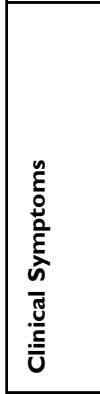 & 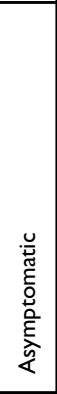 & 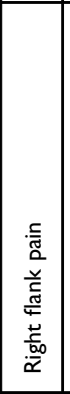 & 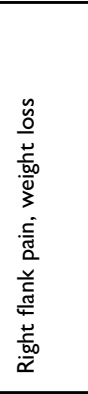 & 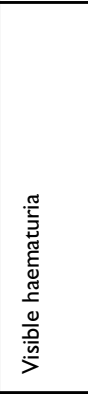 & 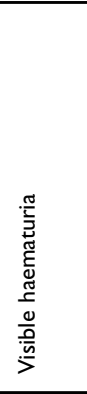 & 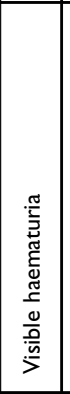 & 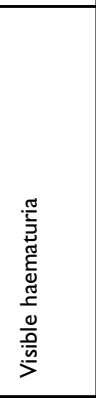 & 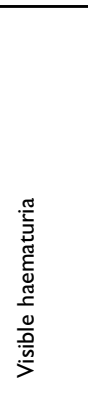 & 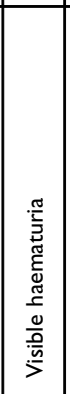 & 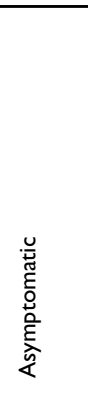 & 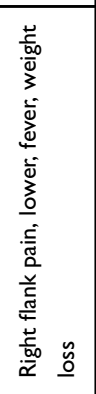 & 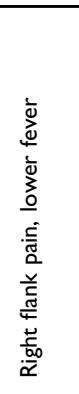 \\
\hline$\overline{\Sigma_{\omega}}$ & $\overline{\bar{g}}$ & $\stackrel{\overline{\dot{N}}}{\bar{N}}$ & $\stackrel{\widehat{S}}{\stackrel{\text { S }}{2}}$ & 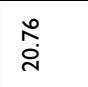 & 㐫 & \begin{tabular}{|c|}
$\substack{\infty \\
\sim}$ \\
$\sim$
\end{tabular} & 足 & 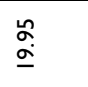 & $\stackrel{\widetilde{\pi}}{\underline{\Xi}}$ & $\stackrel{\alpha}{\alpha}$ & 台 & $\stackrel{\overline{\mathrm{a}}}{\mathrm{i}}$ \\
\hline ع ع & $\frac{0}{\Sigma}$ & \begin{tabular}{|l|}
$\frac{\hat{\sigma}}{\Sigma}$ \\
\end{tabular} & 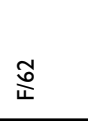 & $\frac{\infty}{\Sigma}$ & $\frac{\mu}{\Sigma}$ & $\frac{\mid \frac{*}{\Sigma}}{\Sigma}$ & $\frac{m}{4}$ & $\frac{\tilde{\rho}}{\Sigma}$ & $\frac{f}{4}$ & $\begin{array}{l}\text { 艿 } \\
\text { 点 }\end{array}$ & $\frac{8}{8}$ & $\frac{\alpha}{\Sigma}$ \\
\hline$\stackrel{9}{\vdots}$ & $\begin{array}{l}\overline{\mathrm{o}} \\
\stackrel{\tilde{g}}{\mathrm{v}}\end{array}$ & \begin{tabular}{|l|} 
\\
$\tilde{g}$ \\
$\tilde{u}$
\end{tabular} & 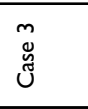 & 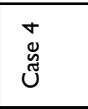 & 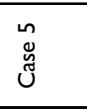 & \begin{tabular}{|l|} 
\\
$\vdots$ \\
$\tilde{o}$ \\
$\tilde{u}$
\end{tabular} & $\begin{array}{l}\widehat{o} \\
\stackrel{\mathscr{m}}{3}\end{array}$ & $\begin{array}{l}\text { o } \\
\text { ơ } \\
\tilde{u}\end{array}$ & $\begin{array}{l}\stackrel{o}{o} \\
\tilde{u} \\
\tilde{u}\end{array}$ & 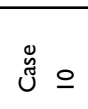 & $\stackrel{\mathscr{ٌ}}{\tilde{J}}=$ & 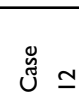 \\
\hline
\end{tabular}



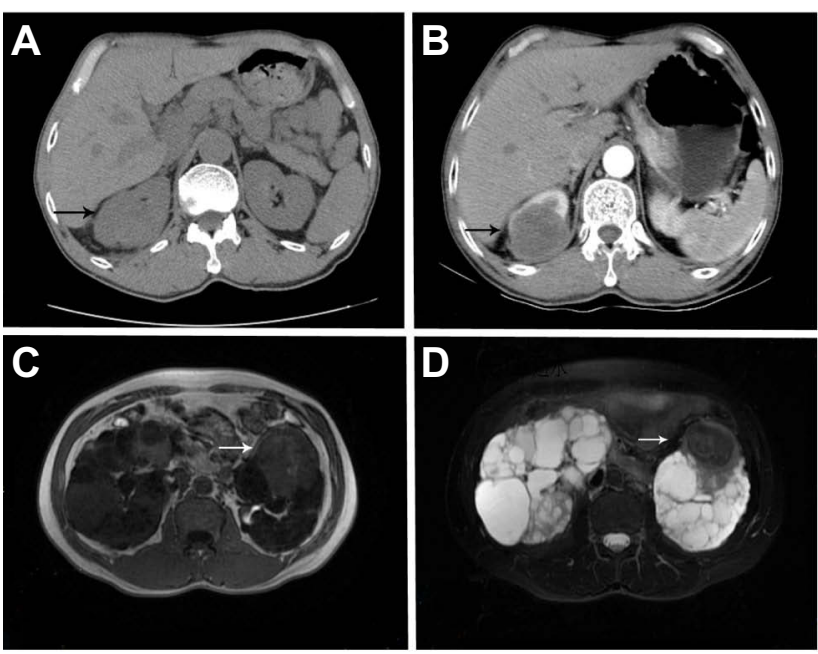

Figure I Case 12. (A) The lesion showed middle density round mass with unclear borders on plain CT scan. And (B) tumor showed lightly and unevenly enhanced around the tumor, and the degree of enhancement was significantly lower than that of peripheral kidney tissues; Case 6. (C) Tumor displayed isointense on TIweighted imaging while (D) a hypointense signal on T2-weighted imaging.

multiple high-density shadows in the kidney, the pyelonephrum dilated slightly, the perifacial fascia thickened and a strip-shaped high-density shadow in front of the kidney were seen on his pain CT scan. Perirenal infection was considered in preoperative diagnosis, but the pathological diagnosis was CDC. On MRI, the tumor showed mixed signal which displayed isointense signal shadows on T1 -weighted images, a shorter signal on T2 -weighted images and had a clear or unclear border, irregular or round-like shapes. Perfusion imaging showed mild to moderate uneven enhancement within the tumor. They were diagnosed as renal neoplastic lesions or malignant tumors of the kidney. And 1 patient with polycystic kidney disease was found (Figure 1). In 1 case, a cystic mixed signal was present in the upper pole lesion of the kidney which was unevenly enhanced, and DWI showed an uneven high signal. An unclear boundary of the kidney calices was seen on the upper pole and finally, he was diagnosed as cystic renal cancer.

\section{Pathology}

Under a light microscope, that collecting ducts or tubules were infiltrated by tumor cells was the typical morphology of CDCs. A tubular, papillary, tubulopapillary (91.7\%) or glandular structures $(83.3 \%)$ or solid architectures $(75 \%)$ with desmoplasia were often presented. And tumor cells had high-grade cytology $(100 \%)$ or an infiltrative growth pattern $(91.7 \%)$. Necrosis of tumor cells also was common in many cases $(33.3 \%)$. And the above features were shown in Figure 2. Besides, tumor cells or other ingredients alike to

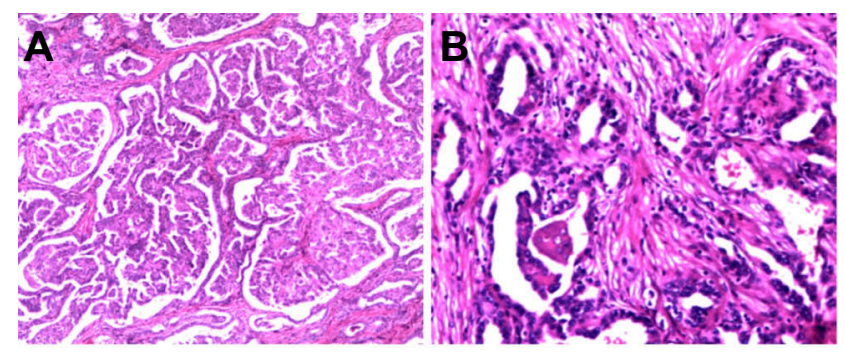

Figure 2 A tubular, papillary growth pattern with desmoplasia and an infiltrative growth pattern were presented. $\square$ A: $\mathrm{HE}, \times 100$; B: $\mathrm{HE}, \times 200$ ).

other typical RCC subtypes of urothelial carcinoma were not discovered. IHC results were expressed as "positive/total numbers". INI-1 (2/2), TFE-3 (2/4), TFE-B (2/3),PCK (9/9), Vimentin (4/9),PAX-8 (5/6), PAX-2 (5/6), CA IX (2/4),CK19 (5/5),EMA (8/8), P504s (5/5), SDHB (3/3),CD117 (1/4),CK7 (5/10), CD10 (3/7), CD15 (1/4), 34 bE12 (4/7), Melan A(0/2), HMB45 (0/3), villin (0/3). And the Ki-67 proliferation index ranged from $3 \%$ to $60 \%$.

\section{Outcomes}

As shown in Table 1, during follow-up, 6/12 (50.0\%) patients had distant metastasis at diagnosis and 5/11 (45.5\%) operative patients had progress. The distant metastatic sites were the lung in 1 patient, thoracic spine (T6-7) in 1 patient, and multiple nodules in the soft tissue, bone, and internal organs in 4 patients. The MST of 11 patients was 11 months. The overall 1-, 3-, and 5-year survival rates were $45.5 \%, 36.4 \%$, and $8.8 \%$, respectively. The survival curve was depicted in Figure 3.

\section{Discussion}

$\mathrm{CDC}$ is a malignant epithelial tumor originating from the distal segment of the renal medullary collecting duct. ${ }^{5}$ It is extremely rare, accounting for about $1 \%$ to $2 \%$ of all types of renal cell carcinoma. ${ }^{6}$ It was FLEMING S. \& LEW H.J.E. who first recognized CDC with high malignancy and rapid progress, which was a distinct pathology subtype of renal cell cancer (RCC). ${ }^{7}$ Most patients had extrarenal metastasis when found and eventually metastasized to lymph nodes, lung, liver, ascites, adrenal gland, and bone., ${ }^{2,5,8}$ In our department, correspondingly, there were about 2200 patients with renal malignant tumors between 2007 and 2017 and CDC only accounted for about $0.5 \%$ of all malignant tumors, which less than $1.0 \%$. And $50.0 \%$ of patients had distant metastasis at diagnosis and $45.5 \%$ of operative patients had progressed. 


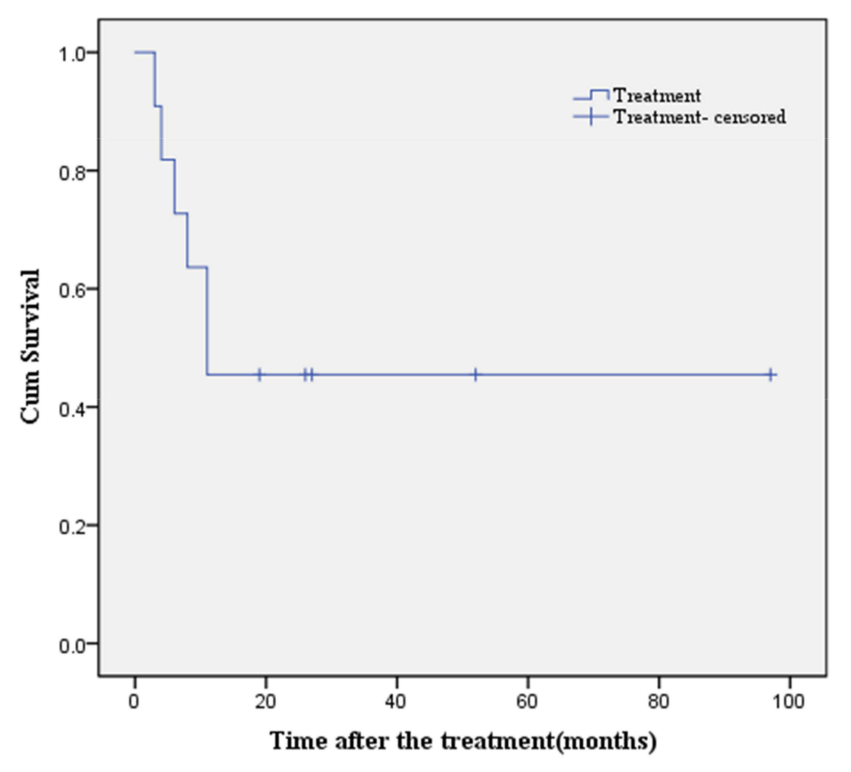

Figure 3 The cumulative probability of survival in patients underwent surgery. Abbreviations: CDC, collecting duct carcinoma; IHC, immunohistochemical staining; CT, computed tomograph; MRI, magnetic resonance imaging; MST, median survival time; RCC, renal cell carcinoma; TNM, tumor node metastasis; VTE, venous thromboembolism; LRN, laparoscopic radical nephrectomy; RN, radical nephrectomy.

CDCs are often found in middle-aged and elderly patients and it has been reported that patients have a median age of 55 years, with more men than women. ${ }^{9}$ In all 12 cases, the male to female ratio was 7:5, consistent with a related report. In previous studies, the median age at diagnosis was 63 years, which did not differ from that for clear cell RCC. ${ }^{10}$ Compared with them, our median age which is 59 years at diagnosis is between 55 and 63 years.

The clinical symptom of the CDC is different, and these differences are related to tumor size, location, invasion, metastasis and so on. Similar to other types of renal malignant tumors, the typical clinical symptoms of patients with CDC often include visible hematuria, abdominal or lumbar pain, fatigue, fever, weight loss, and abdominal masses, but some patients do not show symptoms and find tumors through physical examination. ${ }^{5,8}$ In our group, all patients present various presentations.

Preoperative imaging investigations, including CT or MRI, are a valid approach to recognize CDC, which needs to be verified by postoperative pathology results. CDC originates from the collecting duct of the renal medulla, which infiltrates into the cortical medulla and renal pelvis of the kidney. When the tumor is small, it is usually round or resembles round. It is limited to the renal medulla and the outline of the kidney will not change. These are different from the characteristics of exogenous growth of renal clear cell carcinoma. ${ }^{6,9}$ On CT images, patients with CDC commonly present solitary tumors, medullar location, weak and heterogeneous enhancement, the involvement of the renal sinus, infiltrative growth, preserved renal contour, calcifications, and a cystic component. ${ }^{4,9}$ And about imaging features of MRI of CDC, ZHU and his colleagues discovered that MRI displayed cystic components, undefined masses and that isointense on T1weighted imaging and iso-or hypointense on T2-weighted imaging were seen. Also, enhancement was lower within the CDC than the renal cortex and medulla during all enhanced phases. ${ }^{11}$ Although the study included fewer cases, it is roughly consistent with ours. MRI results also do not present specificity. So, it is difficult to complete the preoperative diagnosis of CDC only by imaging methods. Considering the high degree of malignancy and the poor prognosis of the CDC, when the CDC is suspected on imaging results, surgery is usually used to conduct treatment and to confirm the diagnosis.

That tissues obtained by surgery (RN, NSS, or cytoreductive NS) or percutaneous renal mass biopsy are performed a pathological examination or IHC is the best way to have the right diagnosis. Nevertheless, CDC remains a diagnosis of exclusion combined with supporting diagnostic features. ${ }^{12}$ Features supporting the diagnosis of a CDC include anatomic location (eg collecting duct and renal medullary), tubular/acinar architecture with high-grade cytology, and an infiltrative growth pattern with an associated desmoplastic stroma. ${ }^{5,12}$ The cut surface of CDCs is usually firm, homogeneous, and tan/white/"fleshy" in color. The tumor has an irregular, undefined margin extending into the cortex and often beyond the kidney and has sarcomatoid differentiation in some cases. ${ }^{13-15}$ Origination of some small lesions can be detected by gross evaluation. Architecturally, an acinar/tubular/tubule papillary growth pattern typically predominates, but solid growth, true papillary formation, and satellite nodules may also be presented. Tumor cells often have hobnail nuclei, eosinophilic cytoplasm, and high-grade nuclear features. Necrosis and mitoses of tumor cells also are common. ${ }^{12}$ At the consensus conference, the International Society of Urological Pathology (ISUP) in 2013 agreed that for a diagnosis of CDC to be made, a tumor should show the following features: (1) at least some of the lesion involves the medullary region; (2) there is a predominant formation of tubules; (3) a desmoplastic stromal reaction should be present; (4) cytologic features are high grade; (5) growth pattern is infiltrative; and (6) there is an absence of other typical RCC subtypes 
of urothelial carcinoma. ${ }^{16}$ The main differential diagnosis includes invasive urothelial carcinoma, renal medullary carcinoma, papillary renal cell carcinoma type 2 and unclassified renal cell carcinoma. When it is difficult to distinguish from renal medullary carcinoma, in addition to morphological characteristics, the patient's age, race, history of sickle cell disease or history of hereditary cancers should all be considered. ${ }^{15}$

IHC helps make an accurate differential diagnosis of CDC. Recent definitive diagnostic criteria for CDC required the exclusion of medullary carcinoma (RMC), FH-deficient RCC, urothelial carcinomas of the upper tract, and metastatic carcinomas, and research by Ohe et al recommended that definitive diagnosis of CDC only be made if RMC and FH-deficient renal tumor carcinoma (RCC) are excluded. So it's of great importance to distinguish CDC from RMC and FH-deficient RCC. ${ }^{17}$ High molecular weight cytokeratin, CK19, and 34ßE12 are consistently expressed in tumor cells when vimentin, epithelial membrane antigen, and CD15 are variably expressed. And most transcription factors Pax 2 and Pax 8 have a nuclear reactivity. ${ }^{15}$ PAX8 can the distinct renal origin of the tumor, but renal tumor subtype cannot be told. And urothelial carcinomas of the renal pelvis were up to $23 \%$ in these tumors. ${ }^{17}$ To help confirm renal origin, IHC for PAX8 should be obtained. At the same time, the use of the combination of PAX8, p63, and GATA3 could easily help with distinction. Relevant research demonstrated that GATA3+ or p63+/PAX8 - sensitivity and specificity for urothelial carcinomas were $84 \%$ and $100 \%$, respectively and that the immune profile of PAX $8+/$ p63 supported the diagnosis of CDC with a sensitivity of $85.7 \%$ and a specificity of $100 \%{ }^{18-20}$ Besides, owing to medullary carcinoma having significant morphologic overlap with CDC, the absence of INI-1 may lead to the misdiagnosis of CDC as renal medullary carcinoma in a few patents. And the absence of INI-1 and nuclear expression of OCT3/4 strongly support the diagnosis of renal medullary cancer, not CDC..$^{12,17,21,22}$ Besides, in Ohe et al's cohort, a few cases which were previously diagnosed as CDCs were reclassified as FH-deficient RCCs by using the contemporary markers, $\mathrm{FH}$ and $2 \mathrm{SC}$. In terms of distinguishing $\mathrm{FH}-$ deficient RCCs and CDCs, retained or equivocal $\mathrm{FH}$ expression with negative 2SC staining which was observed in all CDCs can be valuable while complete loss of $\mathrm{FH}$ and induction of $2 \mathrm{SC}$ was seen in all $\mathrm{FH}-$ deficient RCCs. ${ }^{17}$ To ensure the accuracy of diagnosis, the expressions of several common IHC markers, INI-1,
FH, 2SC, PAX-8, p63+, GATA3+ etc, should be tested in therapy. There is a deficiency in our treatment process, that relevant IHC markers should have been tested and diagnosis of our CDCs depended heavily on the typical pathological features, which may result in certain misdiagnosis rates.

CDCs are aggressive malignant tumors, and their prognosis is poor. In our study cohort, the MST of 11 patients was 11 months, and only 1 patient survived for $>5$ years after the diagnosis. Most of the patients having undergone surgery had poor survival. In Abern's study, ${ }^{23}$ patients having accepted treatment (RS, NSS, systemic adjuvant treatment) received the same outcomes. Likely, Sheng et al research manifested that $\mathrm{mCDCs}$ with chemotherapy also presented short progression-free survival (PFS) and overall survival (OS). ${ }^{24}$ Although there is no established treatment, surgery is still the main method of treating the CDC at present, but most patients have discovered lymphatic metastasis or distant metastasis for the first time diagnosis, and whether cytoreductive surgery can benefit patients from survival has not been concluded. There are reports that after surgery patients can improve survival. ${ }^{23}$ In addition, patients with surgical resection of tumors need to be actively followed up. For patients with advanced CDC, other traditional treatments such as chemotherapy, radiation, and immunotherapy can be considered, but the effect is limited to most patients. Considering the cytotoxicity of chemotherapy, the combined use of gemcitabine and platinum salts is considered to treat metastatic CDC. $^{25}$ As a first-line solution, for CDC patients who cannot tolerate surgery and chemotherapy, targeted therapy can be considered as one of the better methods. Some targeted therapy drugs have been used to treat the CDC. The effect needs to be reported by multi-center studies.

\section{Conclusion}

CDCs are extremely rare renal tumors, and they have an aggressive, clinical feature, with a high tendency for distant metastasis and poor survival. The radiographic results of the CDC often have referential value in preoperative diagnosis and differential diagnosis. Accurate diagnosis of this disease depends on the pathological test and IHC. Surgery plays a significant role in the course of therapy of CDCs and chemotherapy, radiation and immunotherapy can be considered, which may benefit patients.

\section{Disclosure}

The authors report no conflicts of interest in this work. 


\section{References}

1. Karakiewicz PI, Trinh Q-D, Rioux-Leclercq N, et al. Collecting duct renal cell carcinoma: a matched analysis of 41 cases. Eur Urol. 2007;52(4):1140-1145. doi:10.1016/j.eururo.2007.01.070

2. Gupta R, Billis A, Shah RB, et al. Carcinoma of the collecting ducts of Bellini and renal medullary carcinoma: clinicopathologic analysis of 52 cases of rare aggressive subtypes of renal cell carcinoma with a focus on their interrelationship. Am J Surg Pathol. 2012;36(9):12651278. doi:10.1097/PAS.0b013e3182635954

3. Tokuda N, Naito S, Matsuzaki O, et al. Collecting duct (bellini duct) renal cell carcinoma: a nationwide survey in Japan. J Urol. 2006;176 (1):40-43. doi:10.1016/S0022-5347(06)00502-7

4. Cuccurullo V, Mansi L. AJCC cancer staging handbook: from the AJCC cancer staging manual (7th edition). Eur J Nucl Med Mol Imaging. 2010;38(2):408. doi:10.1007/s00259-010-1693-9

5. Moch H, Cubilla AL, Humphrey PA, et al. The 2016 WHO classification of tumours of the urinary system and male genital organs-part a: renal, penile, and testicular tumours. Eur Urol. 2016;70(1):93-105. doi:10.1016/j.eururo.2016.02.029

6. Seo AN, Yoon G, Ro JY. Clinicopathologic and molecular pathology of collecting duct carcinoma and related renal cell carcinomas. $A d v$ Anat Pathol. 2017;24(2):65-77. doi:10.1097/PAP.0000000000 000138

7. Fleming S, Lewi HJE. Collecting duct carcinoma of the kidney. Histopathology. 1986;10(11):1131-1141. doi:10.1111/j.1365-2559.19 86.tb02553.x

8. Sui W, Matulay JT, Robins DJ, et al. Collecting duct carcinoma of the kidney: disease characteristics and treatment outcomes from the National Cancer Database. Urol Oncol. 2017;35(9):540 e13-540 e18. doi:10.1016/j.urolonc.2017.04.010

9. Ciszewski S, Jakimow A, Smolska-Ciszewska B. Collecting (Bellini) duct carcinoma: a clinical study of a rare tumour and review of the literature. Can Urol Assoc J. 2015;9(9-10):E589-93. doi:10.5489/ cuaj.2932

10. Dason S, Allard C, Sheridan-Jonah A, et al. Management of renal collecting duct carcinoma: a systematic review and the McMaster experience. Curr Oncol. 2013;20(3):e223-32. doi:10.3747/ co. 20.1230

11. Zhu Q, Wu J, Wang Z, et al. The MSCT and MRI findings of collecting duct carcinoma. Clin Radiol. 2013;68(10):1002-1007. doi:10.1016/j.crad.2013.04.004

12. Hirsch MS, Signoretti S, Dal Cin P. Adult renal cell carcinoma: a review of established entities from morphology to molecular genetics. Surg Pathol Clin. 2015;8(4):587-621. doi:10.1016/j.path.2015. 09.003
13. Rumpelt HJ, Störkel S, Moll R, et al. Bellini duct carcinoma: further evidence for this rare variant of renal cell carcinoma. Histopathology. 1991;18(2):115-122. doi:10.1111/j.1365-2559.1991.tb01453.x

14. Srigley JR, Eble JN. Collecting duct carcinoma of kidney. Semin Diagn Pathol. 1998;15(1):54-67.

15. Fleming S. Distal nephron neoplasms. Semin Diagn Pathol. 2015;32 (2):114-123. doi:10.1053/j.semdp.2015.02.004

16. Srigley JR, Delahunt B, Eble JN, et al. The International Society of Urological Pathology (ISUP) vancouver classification of renal neoplasia. Am J Surg Pathol. 2013;37(10):1469-1489. doi:10.1097/ PAS.0b013e318299f2d1

17. Ohe C, Smith SC, Sirohi D, et al. Reappraisal of morphologic differences between renal medullary carcinoma, collecting duct carcinoma, and fumarate hydratase-deficient renal cell carcinoma. Am J Surg Pathol. 2018;42(3):279-292. doi:10.1097/PAS.00000000 00001000

18. Tong GX, Yu WM, Beaubier NT, et al. Expression of PAX8 in normal and neoplastic renal tissues: an immunohistochemical study. Mod Pathol. 2009;22(9):1218-1227. doi:10.1038/modpathol.2009.88

19. Albadine R, Schultz L, Illei P, et al. PAX8 (+)/p63 (-) immunostaining pattern in renal collecting duct carcinoma (CDC): a useful immunoprofile in the differential diagnosis of CDC versus urothelial carcinoma of upper urinary tract. Am J Surg Pathol. 2010;34 (7):965. doi:10.1097/PAS.0b013e3181dc5e8a

20. Carvalho JC, Thomas DG, McHugh JB, et al. p63, CK7, PAX8 and INI-1: an optimal immunohistochemical panel to distinguish poorly differentiated urothelial cell carcinoma from high-grade tumours of the renal collecting system. Histopathology. 2012;60(4):597-608. doi:10.1111/j.1365-2559.2011.04093.x

21. Elwood H, Chaux A, Schultz L, et al. Immunohistochemical analysis of SMARCB1/INI-1 expression in collecting duct carcinoma. Urology. 2011;78(2):474 e1-5. doi:10.1016/j.urology.2011.04.043

22. Rao P, Tannir NM, Tamboli P. Expression of OCT3/4 in renal medullary carcinoma represents a potential diagnostic pitfall. Am J Surg Pathol. 2012;36(4):583-588. doi:10.1097/PAS.0b013e3182417d78

23. Abern MR, Tsivian M, Polascik TJ, et al. Characteristics and outcomes of tumors arising from the distal nephron. Urology. 2012;80 (1):140-146. doi:10.1016/j.urology.2012.03.034

24. Sheng X, Cao D, Yuan J, et al. Sorafenib in combination with gemcitabine plus cisplatin chemotherapy in metastatic renal collecting duct carcinoma: A prospective, multicentre, single-arm, Phase 2 study. Eur J Cancer. 2018;100:1-7. doi:10.1016/j.ejca.2018.04.007

25. Oudard S, Banu E, Vieillefond A, et al. Prospective multicenter Phase II study of gemcitabine plus platinum salt for metastatic collecting duct carcinoma: results of a GETUG (Groupe d'Etudes des Tumeurs Uro-Genitales) study. $J$ Urol. 2007;177(5):1698-1702. doi:10.1016/j. juro.2007.01.063

\section{Publish your work in this journal}

Cancer Management and Research is an international, peer-reviewed open access journal focusing on cancer research and the optimal use of preventative and integrated treatment interventions to achieve improved outcomes, enhanced survival and quality of life for the cancer patient.
The manuscript management system is completely online and includes a very quick and fair peer-review system, which is all easy to use. Visit http://www.dovepress.com/testimonials.php to read real quotes from published authors. 